\title{
CONSTRAINED PORTFOLIO LIQUIDATION IN A LIMIT ORDER BOOK MODEL
}

\author{
AURÉLIEN ALFONSI \\ CERMICS, projet MATHFI \\ École Nationale des Ponts et Chaussées \\ 6-8 avenue Blaise Pascal, Cité Descartes, Champs sur Marne \\ 77455 Marne-la-Vallée, France \\ E-mail: alfonsi@cermics.enpc.fr \\ ANTJE FRUTH \\ Quantitative Products Laboratory \\ Alexanderstr. 5, 10178 Berlin, Germany \\ E-mail:fruth@math.tu-berlin.de \\ ALEXANDER SCHIED \\ School of ORIE, Cornell University \\ 232 Rhodes Hall, Ithaca, NY 14853, U.S.A. \\ E-mail: schied@cornell.edu
}

\begin{abstract}
We consider the problem of optimally placing market orders so as to minimize the expected liquidity costs from buying a given amount of shares. The liquidity price impact of market orders is described by an extension of a model for a limit order book with resilience that was proposed by Obizhaeva and Wang (2006). We extend their model by allowing for a time-dependent resilience rate, arbitrary trading times, and general equilibrium dynamics for the unaffected bid and ask prices. Our main results solve the problem of minimizing the expected liquidity costs within a given convex set of predictable trading strategies by reducing it to a deterministic optimization problem. This deterministic problem is explicitly solved for the case in which the convex set of strategies is defined via finitely many linear constraints. A detailed study of optimal portfolio liquidation in markets with opening and closing call auctions is provided as
\end{abstract}

2000 Mathematics Subject Classification: 91B26, 91B28, 91B70, 93E20, 60G35.

Key words and phrases: liquidity risk, optimal portfolio liquidation, limit order book with resilience, call auction, market impact model, constrained trading strategies, market order.

Research of the first two authors was supported by Deutsche Forschungsgemeinschaft through the Research Center MATHEON "Mathematics for key technologies" (FZT 86).

The paper is in final form and no version of it will be published elsewhere. 
an illustration. We also obtain closed-form solutions for the unconstrained portfolio liquidation problem in our time-inhomogeneous setting and thus extend a result from our earlier paper [1].

1. Introduction. A common problem for stock traders consists in unwinding large block orders of shares, which can comprise a significant percentage of the daily traded volume of shares. Orders of this size create significant impact on the asset price and, to reduce the overall market impact, it is necessary to split them into smaller orders that are placed during a certain time interval. The question at hand is to allocate an optimal proportion of the entire order to each individual placement so as to minimize the overall price impact. Problems of this type were investigated by Bertsimas and Lo [6], Almgren and Chriss [3, 4], Almgren [2], Almgren and Lorenz [5], Obizhaeva and Wang [13], Schied and Schöneborn [15, 16], and in our earlier paper [1], to mention only a few. For extensions to situations with several competing traders, see [10], [11], [17], and the references therein.

The mathematical formulation of the corresponding optimization problem relies first of all on specifying a stock price model that takes into account the feedback effects resulting from the placement of large orders. It is a well established empirical fact that at least a part of the price impact of large market orders is only temporary. In the majority of models in the literature, this temporary impact has no duration and only instantaneously affects the trade that has triggered it. It is therefore equivalent to a penalization by transaction costs. Models of this type underlie the above-mentioned papers [6], [3], [4], [5], [10], [11], and [17].

Another type of model has recently been proposed by Obizhaeva and Wang [13]. Instead of merely postulating the dynamics of the price process, models of this type derive their dynamics from an intuitive underlying model of a limit order book (LOB). In [13] it is assumed that the ask part of the LOB consists of a uniform distribution of shares offered at prices higher than the current best ask price. When the large trader is not active, the mid price of the LOB fluctuates according to the Bachelier model, and the bid-ask spread remains constant. A buy market order of the large trader consumes a block of shares closest to the best ask and thus increases the ask price proportionally to the size of the order. This corresponds to a linear price impact. As found in empirical studies, the flow of incoming limit sell orders in real-world order books is concentrated in the vicinity of the new best ask price and thus tends to quickly close at least a part of the gap created by the preceding market order; see, for instance, Biais et al. [7], Potters and Bouchaud [14], Bouchaud et al. [9], and Weber and Rosenow [18]. In the LOB model, this resilience effect is described by an exponential decay of the part of the price impact that is not permanent. Hence, the resulting impermanent price impact is neither instantaneous nor entirely permanent but decays on an exponential scale.

In our previous paper [1], we generalized the model from [13] by allowing for a nonlinear shape of the LOB, which leads to a nonlinear price impact. In addition, we considered general equilibrium dynamics for the unaffected bid and ask prices rather than just the Bachelier model. In this extended framework, we obtained closed-form solutions for the problem of minimizing the expected liquidity costs within the class of predictable trading strategies. As a byproduct, we obtained a new closed-form solution for the optimal 
strategy in a block-shaped LOB, whereas in [13] this strategy was only given in terms of an entangled forward-backward recursive scheme.

In this paper, we look at a different extension of the market impact model from [13]. It is well known to practitioners that trading volume and liquidity are inhomogeneously distributed over the trading day, often with a U-shaped or W-shaped pattern. Due to the mechanism of LOB recovery described above, one thus expects a similar timeinhomogeneous pattern for the resilience rate of the LOB. Therefore, we allow for a time-dependent resilience rate in our model and, as a consequence, are also able to treat arbitrarily spaced trading times. On the other hand, we use a block-shaped LOB as in $[13]$.

After introducing the time-inhomogeneous LOB model, we consider the problem of optimally executing a buy order for $X_{0}$ shares within a certain time frame $[0, T]$. The focus on buy orders is for the simplicity of the presentation only; completely analogous results hold for sell orders as well. While most other papers, including [13], focus on optimization within the class of deterministic strategies, we will here allow for dynamic updating of trading strategies, that is, we optimize over the larger class of adapted strategies. We also allow for intermediate sell orders in our strategies.

Our first result, Theorem 3.1, provides an explicit solution of the optimal portfolio liquidation problem in our time-inhomogeneous framework and thus extends [1, Corollary 6.1]. Our main contribution, however, is to analyze the cost optimization problem under convex constraints on our trading strategies. Such constraints can arise rather naturally in many situations. For instance, linear constraints will allow us to give a detailed analysis of optimal liquidation strategies in markets with opening and closing call auctions.

In a first step to solving the optimal portfolio liquidation problem, Theorem 3.3 reduces the original constrained stochastic optimization problem to the minimization of a positive definite quadratic form on a convex subset of Euclidean space. We are then able to explicitly compute the inverse of the corresponding matrix and thus, in a second step, to solve the finite-dimensional minimization problem by means of the Kuhn-Tucker theorem. The corresponding result is formulated in Theorem 3.4, together with an explicit solution for the case of finitely many linear constraints.

The paper is organized as follows. In Section 2, we explain the market impact model based on LOB dynamics. In Section 3, we set up the resulting optimization problems and state our main results. The case study of a market with opening and closing call auctions is carried out in Section 4. The proofs of our main results are given in Sections 5 and 6.

2. Model setup. In this section, we present an extension of the market impact model of Obizhaeva and Wang [13]. This one-asset model derives its price dynamics from a dynamic model of a limit order book (LOB) with resilience. A limit order is an order to buy or sell the asset at a specified price, and a LOB consists of the collection of all buy and sell limit orders at various prices. Matching buy and sell limit orders are immediately executed, so a limit order book consists of two disjoint blocks of buy and sell limit orders. The lowest price asked for a sell limit order is called the best ask price. It is larger than 
the highest bid for buying shares, which is usually called the best bid price. Following [13], we here make the simplifying assumption that the ask and bid sides of the LOB consist of a continuous price distribution of orders with constant height $q>0$. That is, for $h>0$, $q \cdot h$ is the amount of shares offered in a price interval $[x, x+h]$ for some price $x \in \mathbb{R}$ that is bigger than the best ask price. Similarly, if $x \in \mathbb{R}$ is lower than the best bid price, then $q \cdot h$ is the amount of buy limit orders in the interval $[x-h, x]$; see Figure 1.

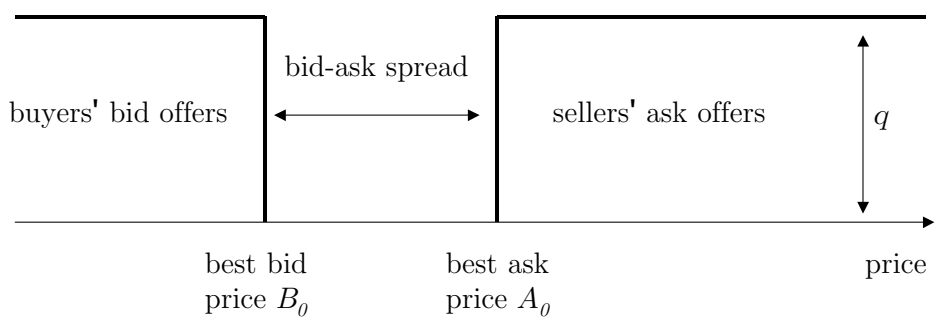

Fig. 1. The limit order book model before the large investor is active

We now consider the actions of a large trader whose goal is to purchase a large amount $X_{0}>0$ of shares within a certain time period $[0, T]$. In reality, $T$ typically ranges from a few hours up to a few trading days. Since the timing of orders is critical, the large trader uses market orders rather than limit orders. A market order consists of an order to buy (or sell) a specific number of shares at the best price currently available. Thus, a market buy order of $x_{0}>0$ shares placed at time $t=0$ consumes all shares between the current best ask price, $A_{0}$, and the price $A_{0+}$ that is determined by the condition $q \cdot\left(A_{0+}-A_{0}\right)=x_{0}$. Thus, $A_{0+}$ will be the best ask price immediately after the execution of the market order; see Figure 2.

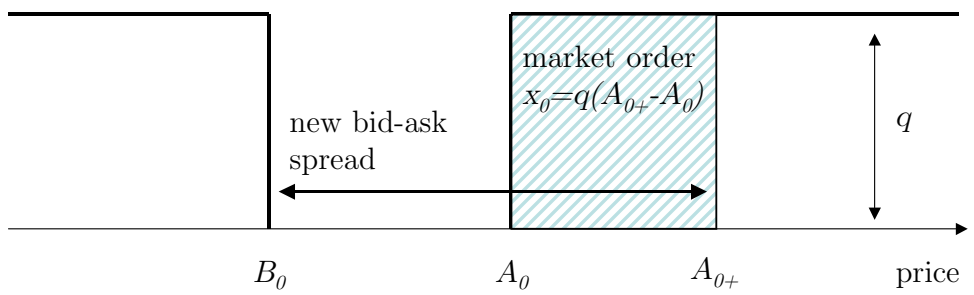

Fig. 2. Impact of a market buy order of $x_{0}$ shares

To describe the dynamics of the LOB between the recurrent orders of the large trader, it is convenient to first specify reference dynamics for the case when the large trader is never active. In this case, the dynamics of the limit order book are determined by the actions of noise traders only. We assume that the corresponding unaffected best ask price, $A_{t}^{0}, t \geq 0$, is a martingale on a given filtered probability space $\left(\Omega,\left(\mathcal{F}_{t}\right), \mathcal{F}, \mathbb{P}\right)$ and satisfies $A_{0}^{0}=A_{0}$. This assumption includes in particular the case in which $A^{0}$ is a Bachelier model, i.e., $A_{t}^{0}=A_{0}+\sigma W_{t}$ for an $\left(\mathcal{F}_{t}\right)$-Brownian motion $W$, as considered 
in [13]. We emphasize, however, that we can take any martingale and hence use, e.g., a risk-neutral geometric Brownian motion, which avoids the counterintuitive negative prices of the Bachelier model. Moreover, we can allow for jumps in the dynamics of $A^{0}$ so as to model the trading activities of other large traders in the market.

When the large trader is active, his or her previous market orders will have moved the best ask price away from the unaffected best ask price $A_{t}^{0}$. Let us thus denote by $A_{t}$ the actual best ask price at time $t$, and let

$$
D_{t}^{A}:=A_{t}-A_{t}^{0}
$$

be the extra spread caused by the past transactions of the trader. Another buy market order of $x_{t}>0$ shares placed at time $t$ consumes all the shares offered at prices between $A_{t}$ and

$$
A_{t+}:=A_{t}+D_{t+}^{A}-D_{t}^{A}=A_{t}^{0}+D_{t+}^{A},
$$

where $D_{t+}^{A}$ is determined by the condition

$$
\left(D_{t+}^{A}-D_{t}^{A}\right) \cdot q=x_{t} .
$$

Thus, the process $D^{A}$ captures the impact of market orders on the current best ask price.

We still need to specify how $D^{A}$ evolves when our trader is inactive in between market orders. It is a well established empirical fact that order books exhibit a certain resilience as to the price impact of large block orders; see, e.g., Biais et al. [7], Potters and Bouchaud [14], Bouchaud et al. [9], and Weber and Rosenow [18]. That is, only a fraction of the immediate impact $x_{t} / q$ is permanent, while the remaining fraction is impermanent and decays to zero. In modeling this resilience, we consider an exponential recovery of the impermanent part of the impact with a time-dependent recovery rate $\rho_{t}$, which we assume to be a strictly positive measurable function on $[0, T]$. More precisely, the price impact at time $t+u$ of a buy market order $x_{t}$ placed at time $t$ is supposed to be

$$
\gamma x_{t}+\kappa e^{-\int_{t}^{t+u} \rho_{s} d s} x_{t}
$$

where $\gamma<1 / q$ quantifies the fraction of the permanent impact and

$$
\kappa:=\frac{1}{q}-\gamma
$$

is the portion of the impermanent impact. The process $D^{A}$ is now defined as the cumulative impact of all past buy market orders. That is, if buy market orders $x_{t_{n}}$ are placed at times $t_{n}$, then

$$
D_{t}^{A}=\gamma \sum_{t_{n}<t} x_{t_{n}}+\kappa \sum_{t_{n}<t} e^{-\int_{t_{n}}^{t} \rho_{s} d s} x_{t_{n}} .
$$

Such an extension of their original model was suggest by Obizhaeva and Wang in [13, Section 8.1].

Up to now, we have only described the effect of buy market orders on the upper half of the LOB. Since the overall goal of the trader is to buy $X_{0}>0$ shares up to time $T$, a restriction to buy orders would seem to be plausible. However, we do not wish to exclude the a priori possibility that, under certain market conditions, it could be beneficial to also sell some shares and to buy them back at a later point in time. To incorporate this 
possibility, we also need to model the impact of sell market orders on the lower part of the LOB. As for ask prices, we will distinguish between an unaffected best bid price, $B_{t}^{0}$, and the actual best bid price, $B_{t}$, for which the price impact of previous sell orders of our trader is taken into account. All we assume on the dynamics of $B^{0}$ is

$$
B_{t}^{0} \leq A_{t} \quad \text { at all times } t .
$$

This assumption allows the unaffected best bid price $B_{t}^{0}$ to depend in a nontrivial way on past buy orders of the trader, which for large buy orders is quite realistic. We do not need to specify the details of this interaction, however. The asymmetry in assumptions on the dynamics of $A^{0}$ and $B^{0}$ is natural in view of the overall task of the trader, which consists in buying a large amount of shares.

The quantity

$$
D_{t}^{B}:=B_{t}-B_{t}^{0}
$$

is called the extra spread in the bid price. A sell market order of $x_{t}<0$ shares placed at time $t$ will consume all the shares offered at prices between $B_{t}$ and

$$
B_{t+}:=B_{t}+D_{t+}^{B}-D_{t}^{B}=B_{t}^{0}+D_{t+}^{B},
$$

where $D_{t+}^{B}$ is determined by the condition $\left(D_{t+}^{B}-D_{t}^{B}\right) \cdot q=x_{t}$. More precisely, we assume that the process $D^{B}$ behaves analogously to its counterpart $D^{A}$, i.e.,

$$
D_{t}^{B}=\gamma \sum_{t_{n}<t} x_{t_{n}}+\kappa \sum_{t_{n}<t} e^{-\int_{t_{n}}^{t} \rho_{s} d s} x_{t_{n}},
$$

where the sum is taken over all past sell market orders $x_{t_{n}}$.

3. The cost minimization problem. When placing a single buy market order of size $x_{t} \geq 0$ at time $t$, the trader purchases $q d x$ shares at price $A_{t}^{0}+x$, with $x$ ranging from $D_{t}^{A}$ to $D_{t+}^{A}$. Hence, the total cost of the buy market order amounts to

$$
\pi_{t}\left(x_{t}\right):=\int_{D_{t}^{A}}^{D_{t+}^{A}}\left(A_{t}^{0}+x\right) q d x=A_{t}^{0} x_{t}+\frac{q}{2}\left(\left(D_{t+}^{A}\right)^{2}-\left(D_{t}^{A}\right)^{2}\right) .
$$

For a sell market order $x_{t} \leq 0$, we have

$$
\pi_{t}\left(x_{t}\right):=B_{t}^{0} x_{t}+\frac{q}{2}\left(\left(D_{t+}^{B}\right)^{2}-\left(D_{t}^{B}\right)^{2}\right) .
$$

In practice, very large orders are often split into a number of consecutive market orders to reduce the overall price impact. Hence, the question at hand is to determine the size of the individual orders so as to minimize a cost criterion. So let us assume that the trader needs to buy a total of $X_{0}>0$ shares until time $T$ and that trading can occur at $N+1$ predetermined times $0 \leq t_{0}<t_{1}<\cdots<t_{N} \leq T$. We emphasize that we do not require these times to be located at equidistant points as is assumed in [13] and [1]. An admissible strategy is a sequence $\xi=\left(\xi_{0}, \xi_{1}, \ldots, \xi_{N}\right)$ of random variables such that

- $\sum_{n=0}^{N} \xi_{n}=X_{0}$,

- each $\xi_{n}$ is measurable with respect to $\mathcal{F}_{t_{n}}$,

- each $\xi_{n}$ is bounded from below. 
The quantity $\xi_{n}$ corresponds to the size of the market order placed at time $t_{n}$. Note that we do not a priori require $\xi_{n}$ to be positive, i.e., we also allow for intermediate sell orders, but we make the realistic assumption that there is some lower bound on sell orders.

The average cost $\mathcal{C}(\xi)$ of an admissible strategy $\xi$ is defined as the expected value of the total costs incurred by the consecutive market orders:

$$
\mathcal{C}(\xi)=\mathbb{E}\left[\sum_{n=0}^{N} \pi_{t_{n}}\left(\xi_{n}\right)\right] .
$$

Our first goal in this paper consists in finding admissible strategies that minimize the average cost within the class of all admissible strategies. The corresponding result can be found in the next theorem. It provides an explicit solution for the time-inhomogeneous version of the forward-backward recursive scheme of [13, Proposition 1] and thus generalizes [1, Corollary 6.1]. Subsequently, we will consider the problem of minimizing the cost functional $\mathcal{C}$ under additional constraints on strategies. Let us introduce the following notation:

$$
a_{0}:=0 \quad \text { and } \quad a_{n}:=e^{-\int_{t_{n-1}}^{t_{n}} \rho_{s} d s} \quad \text { for } n=1, \ldots, N .
$$

THEOREM 3.1 (Unconstrained optimal strategy). There exists a unique optimal strategy $\xi^{*}=\left(\xi_{0}^{*}, \ldots, \xi_{N}^{*}\right)$ in the class of all admissible strategies. With the notation

$$
\lambda_{0}:=\frac{X_{0}}{\frac{2}{1+a_{1}}+\sum_{n=2}^{N} \frac{1-a_{n}}{1+a_{n}}},
$$

the initial market order is

$$
\xi_{0}^{*}=\frac{\lambda_{0}}{1+a_{1}},
$$

the intermediate market orders are given by

$$
\xi_{n}^{*}=\lambda_{0}\left(\frac{1}{1+a_{n}}-\frac{a_{n+1}}{1+a_{n+1}}\right), \quad n=1, \ldots, N-1,
$$

and the final market order is

$$
\xi_{N}^{*}=\frac{\lambda_{0}}{1+a_{N}} .
$$

In particular, the optimal strategy is deterministic. Moreover, it consists only of nontrivial buy orders, i.e., $\xi_{n}^{*}>0$ for all $n$.

An important feature of our method is that it can easily be extended to incorporate constraints on strategies. In fact, Theorem 3.1 is a special case of our more general results. Its proof will be derived from these general results in Example 3.5.

REMARK 3.2 (Time-homogeneous case). Let us consider the case of a constant resilience speed $\rho>0$ and an equidistant time grid $t_{n}=n T / N, n=0, \ldots, N$, as considered by Obizhaeva and Wang [13] and Section 6 of our earlier paper [1]. Then $a_{n}=e^{-\rho T / N}=: a$, and the optimal strategy becomes

$$
\xi_{0}^{*}=\xi_{N}^{*}=\frac{X_{0}}{(N-1)(1-a)+2} \quad \text { and } \quad \xi_{1}^{*}=\cdots=\xi_{N-1}^{*}=\xi_{0}^{*}(1-a) .
$$


This is equivalent to our formula given in [1, Corollary 6.1]. Thus, the proof of our Theorem 3.1 provides a new approach to the optimal execution problem in [13]. While it provides the same explicit solution as in our earlier paper [1], the proof given here is significantly shorter and simpler in comparison. Note that the solution in [13] is only given in terms of an entangled forward-backward recursion formula and that our setup differs by allowing for general martingale dynamics of $A^{0}$ and for a larger class of admissible strategies.

To formulate our general results on optimal portfolio liquidation under constraints, let us first introduce some notation. With 1 we will denote the $(N+1)$-dimensional column vector $(1, \ldots, 1)^{\prime}$, where the apostrophe indicates the transpose of a vector or a matrix. An admissible strategy can be regarded as a measurable map $\xi: \Omega \rightarrow \mathbb{R}^{N+1}$ such that the inner product with $\mathbf{1}$ satisfies $\langle\xi, \mathbf{1}\rangle=X_{0}$. We thus introduce

$$
\Xi:=\left\{\mathbf{x} \in \mathbb{R}^{N+1} \mid\langle\mathbf{x}, \mathbf{1}\rangle=X_{0}\right\} .
$$

Constraints on strategies can now be modeled by considering only admissible strategies $\xi$ such that $\xi: \Omega \rightarrow \Xi_{0}$, where $\Xi_{0}$ is a closed convex subset of $\Xi$. We have the following abstract result on constrained portfolio liquidation, which reduces the original cost optimization problem to the minimization of the quadratic form corresponding to the symmetric matrix

$$
M:=\left[\begin{array}{cccccc}
1 & a_{1} & a_{1} a_{2} & \ldots & \cdots & a_{1} a_{2} \cdots a_{N} \\
a_{1} & 1 & a_{2} & a_{2} a_{3} & \ldots & a_{2} a_{3} \cdots a_{N} \\
a_{1} a_{2} & a_{2} & 1 & a_{3} & \ldots & \vdots \\
\vdots & & \ddots & \ddots & \ddots & \vdots \\
a_{2} \cdots a_{N} & & & a_{N-1} & 1 & a_{N} \\
a_{1} a_{2} \cdots a_{N} & \cdots & \cdots & a_{N-1} a_{N} & a_{N} & 1
\end{array}\right] .
$$

Theorem 3.3 (Reduction to a deterministic problem). Let $\Xi_{0}$ be a closed convex subset of $\Xi$. Then the following assertions hold.

(a) The matrix $M$ is positive definite, and so the quadratic form

$$
C(\mathbf{x})=\frac{1}{2}\langle\mathbf{x}, M \mathbf{x}\rangle, \quad \mathbf{x} \in \Xi_{0},
$$

admits a unique minimizer $\mathbf{x}^{*}=\left(x_{0}^{*}, \ldots, x_{N}^{*}\right)$ in $\Xi_{0}$.

(b) If $x_{n}^{*} \geq 0$ for $n=0, \ldots, N$, then $\xi_{n}^{*}:=x_{n}^{*}$ is the unique optimal liquidation strategy within the class of $\Xi_{0}$-valued admissible strategies.

Given the preceding theorem, we focus now on the constrained minimization of the quadratic form $C(\mathbf{x})=\frac{1}{2}\langle\mathbf{x}, M \mathbf{x}\rangle$. This can be done by applying the Kuhn-Tucker theorem. While this approach can, in principle, be carried out for rather general, nonlinear constraints, it will often be sufficient to work with linear constraints in practical applications. Confinement to linear constraints also greatly simplifies the complexity of the problem. Let us now formulate a corresponding result. We assume that the linear constraints on strategies are defined via vectors $\mathbf{u}^{1}, \ldots, \mathbf{u}^{k}, \mathbf{v}^{1}, \ldots, \mathbf{v}^{l} \in \mathbb{R}^{N+1}$, where 
$k, l \in \mathbb{N}_{0}$, and we consider the following constraints set:

$$
\Xi_{0}=\left\{\mathbf{x} \in \Xi \mid\left\langle\mathbf{u}^{i}, \mathbf{x}\right\rangle=0, i=1, \ldots, k,\left\langle\mathbf{v}^{j}, \mathbf{x}\right\rangle \geq 0, j=1, \ldots, l\right\} .
$$

For instance, the constraint $x_{0}+x_{N} \geq X_{0} / 4$ can be obtained as $\langle\mathbf{v}, \mathbf{x}\rangle \geq 0$ for $\mathbf{v}=$ $(1,0, \ldots, 0,1)^{\prime}-\frac{1}{4} \mathbf{1}$.

THEOREM 3.4 (Solution of the deterministic problem). Let $\mathbf{x}^{*}$ be the unique minimizer of $C(\mathbf{x})=\frac{1}{2}\langle\mathbf{x}, M \mathbf{x}\rangle$ on the set $\Xi_{0}$ defined in (12), and let $J$ denote the index set of active constraints for $\mathbf{x}^{*}$, that is, the set of all $j \in\{1, \ldots, l\}$ such that $\left\langle\mathbf{v}^{j}, \mathbf{x}^{*}\right\rangle=0$. Suppose furthermore that $\{\mathbf{1}\} \cup\left\{\mathbf{u}^{1}, \ldots, \mathbf{u}^{k}\right\} \cup\left\{\mathbf{v}^{j} \mid j \in J\right\}$ is a set of linearly independent vectors in $\mathbb{R}^{N+1}$. Then

$$
\mathbf{x}^{*}=\lambda_{0} M^{-1} \mathbf{1}+\sum_{i=1}^{k} \lambda_{i} M^{-1} \mathbf{u}^{i}+\sum_{j \in J} \mu_{j} M^{-1} \mathbf{v}^{j}
$$

for multipliers $\lambda_{0}, \lambda_{1}, \ldots, \lambda_{k} \in \mathbb{R}$ and $\mu_{j} \geq 0, j \in J$, which are uniquely determined by the following system of linear equations:

$$
\begin{aligned}
X_{0} & =\lambda_{0}\left\langle\mathbf{1}, M^{-1} \mathbf{1}\right\rangle+\sum_{i=1}^{k} \lambda_{i}\left\langle\mathbf{1}, M^{-1} \mathbf{u}^{i}\right\rangle+\sum_{j \in J} \mu_{j}\left\langle\mathbf{1}, M^{-1} \mathbf{v}^{j}\right\rangle \\
0 & =\lambda_{0}\left\langle\mathbf{u}^{p}, M^{-1} \mathbf{1}\right\rangle+\sum_{i=1}^{k} \lambda_{i}\left\langle\mathbf{u}^{p}, M^{-1} \mathbf{u}^{i}\right\rangle+\sum_{j \in J} \mu_{j}\left\langle\mathbf{u}^{p}, M^{-1} \mathbf{v}^{j}\right\rangle \\
0 & =\lambda_{0}\left\langle\mathbf{v}^{q}, M^{-1} \mathbf{1}\right\rangle+\sum_{i=1}^{k} \lambda_{i}\left\langle\mathbf{v}^{q}, M^{-1} \mathbf{u}^{i}\right\rangle+\sum_{j \in J} \mu_{j}\left\langle\mathbf{v}^{q}, M^{-1} \mathbf{v}^{j}\right\rangle,
\end{aligned}
$$

where $p$ runs through $\{1, \ldots, k\}$ and $q$ through $J$.

Moreover, the inverse of $M$ is explicitly given as the tridiagonal matrix

$$
M^{-1}=\left[\begin{array}{ccccc}
\frac{1}{1-a_{1}^{2}} & \frac{-a_{1}}{1-a_{1}^{2}} & 0 & \cdots & 0 \\
\frac{-a_{1}}{1-a_{1}^{2}} & \left(\frac{1}{1-a_{1}^{2}}+\frac{a_{2}^{2}}{1-a_{2}^{2}}\right) & \frac{-a_{2}}{1-a_{2}^{2}} & 0 \cdots & 0 \\
0 & \ddots & \ddots & \ddots & \vdots \\
\vdots & \ddots & \frac{-a_{N-1}}{1-a_{N-1}^{2}} & \left(\frac{1}{1-a_{N-1}^{2}}+\frac{a_{N}^{2}}{1-a_{N}^{2}}\right) & \frac{-a_{N}}{1-a_{N}^{2}} \\
0 & \cdots & 0 & \frac{-a_{N}}{1-a_{N}^{2}} & \frac{1}{1-a_{N}^{2}}
\end{array}\right] .
$$

We now discuss examples and applications illustrating the use of Theorems 3.3 and 3.4. The first application is actually the proof of Theorem 3.1.

EXAMPLE 3.5 (Proof of Theorem 3.1). In the unconstrained case we have $\Xi_{0}=\Xi$, and so (13) reduces to $\mathbf{x}^{*}=\lambda_{0} M^{-1} \mathbf{1}$ and (14) to $\lambda_{0}=X_{0} /\left\langle\mathbf{1}, M^{-1} \mathbf{1}\right\rangle$. One easily shows that

$$
M^{-1} \mathbf{1}=\left[\begin{array}{c}
\frac{1}{1+a_{1}} \\
\frac{1}{1+a_{1}}-\frac{a_{2}}{1+a_{2}} \\
\vdots \\
\frac{1}{1+a_{N-1}}-\frac{a_{N}}{1+a_{N}} \\
\frac{1}{1+a_{N}}
\end{array}\right] \text {, }
$$


and this gives

$$
\lambda_{0}=\frac{X_{0}}{\frac{2}{1+a_{1}}+\sum_{n=2}^{N} \frac{1-a_{n}}{1+a_{n}}} .
$$

From this we obtain the formula of the optimal strategy in Theorem 3.1. We still need to show that all components of $\mathbf{x}^{*}=\lambda_{0} M^{-1} \mathbf{1}=\left(x_{0}^{*}, \ldots, x_{N}^{*}\right)$ are strictly positive. This is clear for $x_{0}^{*}$ and $x_{N}^{*}$ since $0<a_{n}<1$ for $n=1, \ldots, N$. For $i=1, \ldots, N-1$ we have

$$
x_{i}^{*}=\lambda_{0} \cdot \frac{\left(1-a_{i} a_{i+1}\right)}{\left(1+a_{i}\right)\left(1+a_{i+1}\right)}>0 .
$$

This, together with an application of Theorem 3.3, concludes the proof of Theorem 3.1.

One can think of a number of applications and examples for constraints in portfolio liquidation. For instance, in view of additional volatility risk it may be preferable to buy faster and thus require $\left\langle\mathbf{v}, \mathbf{x}^{*}\right\rangle \geq 0$ for some vector $\mathbf{v}$ with decreasing components. In the next section, we give a detailed case study dealing with a possible approach to portfolio liquidation in stock markets with call auctions.

4. Liquidation strategies in markets with call auctions. At most stock exchanges, morning and evening call auctions take place at the beginning and end of every trading day. Continuous trading is halted during the auction period, while bidding continues. As a result, the sell and buy sides of the order book may start overlapping. At the end of this so-called order collection or calling phase, a price per share is determined so that the overall volume of executed trades is maximized. Although the duration of an auction is usually in the order of a few minutes, a significant amount of shares may be traded within this period. For instance, Kehr et al. [12] investigate data from stocks in the DAX index for the year 1996 and find that more than 20\% of the daily traded Siemens shares are exchanged during auctions.

In view of the huge trading volume during auctions, traders may want to make sure that, in their liquidation strategies, a minimum percentage of shares is traded during the auctions. To illustrate a possible approach, let us suppose that $[0, T]$ is one trading day and that the first and last market orders occur during the respective opening and closing auctions. Hence there is one trade per auction. To make sure that at least $\alpha \times 100$ percent of shares are traded during these auctions, the trader may want to impose the constraint $\xi_{0}+\xi_{N} \geq \alpha X_{0}$ for some $\left.\alpha \in\right] 0,1\left[\right.$. The corresponding set $\Xi_{0}$ is given as

$$
\Xi_{0}=\{\mathbf{x} \in \Xi \mid\langle\mathbf{v}, \mathbf{x}\rangle \geq 0\} \quad \text { for } \quad \mathbf{v}=(1,0, \ldots, 0,1)^{\prime}-\alpha \mathbf{1} .
$$

Clearly, $\mathbf{1}$ and $\mathbf{v}$ are linearly independent, so the assumptions of Theorem 3.4 are automatically satisfied. The minimizer $\mathbf{x}^{*}$ is therefore given by

$$
\mathbf{x}^{*}=\lambda_{0} M^{-1} \mathbf{1}+\mu M^{-1} \mathbf{v}
$$

for certain multipliers $\lambda_{0} \in \mathbb{R}$ and $\mu \geq 0$. The constraint $\langle\mathbf{x}, \mathbf{v}\rangle \geq 0$ is only nontrivial if $\alpha X_{0}$ is larger than the sum of the first and last market orders of the unconstrained optimal strategy from Theorem 3.1. In this case, we must have $\left\langle\mathbf{x}^{*}, \mathbf{v}\right\rangle=0$ and (14) 
becomes

$$
\begin{aligned}
X_{0} & =\lambda_{0}\left\langle\mathbf{1}, M^{-1} \mathbf{1}\right\rangle+\mu\left\langle\mathbf{1}, M^{-1} \mathbf{v}\right\rangle, \\
0 & =\lambda_{0}\left\langle\mathbf{v}, M^{-1} \mathbf{1}\right\rangle+\mu\left\langle\mathbf{v}, M^{-1} \mathbf{v}\right\rangle .
\end{aligned}
$$

The symmetry of $M^{-1}$ implies $\left\langle\mathbf{1}, M^{-1} \mathbf{v}\right\rangle=\left\langle\mathbf{v}, M^{-1} \mathbf{1}\right\rangle$, and so the values for $\lambda_{0}$ and $\mu$ are obtained as

$$
\lambda_{0}=C\left\langle\mathbf{v}, M^{-1} \mathbf{v}\right\rangle \quad \text { and } \quad \mu=-C\left\langle\mathbf{v}, M^{-1} \mathbf{1}\right\rangle
$$

for

$$
C:=\frac{X_{0}}{\left\langle\mathbf{1}, M^{-1} \mathbf{1}\right\rangle\left\langle\mathbf{v}, M^{-1} \mathbf{v}\right\rangle-\left\langle\mathbf{v}, M^{-1} \mathbf{1}\right\rangle^{2}} .
$$

Note that $0<C<\infty$ since $M$ is positive definite. We see in particular that the condition $\mu \geq 0$ is equivalent to $\left\langle\mathbf{v}, M^{-1} \mathbf{1}\right\rangle \leq 0$. By Example $3.5, M^{-1} \mathbf{1}$ is proportional to the optimal strategy $\xi^{*}$ in Theorem 3.1. Therefore $\mu \geq 0$ holds if and only if $\xi^{*}$ does not already satisfy $\xi_{0}^{*}+\xi_{N}^{*}>\alpha X_{0}$.

Using the shorthand notation $\mathbf{w}:=(1,0 \ldots, 0,1)^{\prime}$, we thus get

$$
\mathbf{x}^{*}=C\left(\left\langle\mathbf{v}, M^{-1} \mathbf{w}\right\rangle M^{-1} \mathbf{1}-\left\langle\mathbf{v}, M^{-1} \mathbf{1}\right\rangle M^{-1} \mathbf{w}\right) .
$$

To continue, we make the simplifying assumption that resilience during auctions is such that $a_{1}=a_{N}=a$, while for regular trading $a_{2}=\cdots=a_{N-1}=b$. We then have

$$
M^{-1} \mathbf{w}=\frac{1}{1-a^{2}} \cdot(1,-a, 0, \ldots, 0,-a, 1)^{\prime},
$$

and hence, by using (16),

$$
\begin{aligned}
\left\langle\mathbf{w}, M^{-1} \mathbf{w}\right\rangle & =\frac{2}{1-a^{2}}, \\
\left\langle\mathbf{1}, M^{-1} \mathbf{w}\right\rangle=\left\langle\mathbf{w}, M^{-1} \mathbf{1}\right\rangle & =\frac{2}{1+a}, \\
\left\langle\mathbf{1}, M^{-1} \mathbf{1}\right\rangle & =\frac{3-a}{1+a}+(N-2) \frac{1-b}{1+b}, \\
\left\langle\mathbf{v}, M^{-1} \mathbf{w}\right\rangle & =2 \cdot \frac{1-\alpha(1-a)}{1-a^{2}}, \\
\left\langle\mathbf{v}, M^{-1} \mathbf{1}\right\rangle & =\frac{2-\alpha(3-a)}{1+a}-\alpha(N-2) \frac{1-b}{1+b} .
\end{aligned}
$$

Now we can compute the optimal strategy. It is clear a priori that $x_{0}^{*}+x_{N}^{*}=\alpha X_{0}$. Since moreover $x_{0}^{*}=x_{N}^{*}$, we have

$$
x_{0}^{*}=x_{N}^{*}=\frac{\alpha X_{0}}{2} .
$$

On the other hand, the formulas above give us

$$
x_{0}^{*}=x_{N}^{*}=\frac{\alpha C}{1-a^{2}}\left(1+(N-2) \frac{1-b}{1+b}\right),
$$

and this yields the following simple formula for $C$ :

$$
C=X_{0} \cdot \frac{1-a^{2}}{2\left(1+(N-2) \frac{1-b}{1+b}\right)} .
$$


Consequently,

$$
x_{2}^{*}=\cdots=x_{N-2}^{*}=X_{0} \cdot \frac{(1-\alpha(1-a))(1-b)}{N-1-(N-3) b},
$$

which is positive for any choice of $\alpha$. Finally,

$$
x_{1}^{*}=x_{N-1}^{*}=X_{0} \cdot \frac{1-\alpha\left(\frac{1}{2} a(1-b)(N-3)+1\right)}{N-1-(N-3) b} .
$$

Hence, if

$$
\alpha\left(\frac{1}{2} a(1-b)(N-3)+1\right) \leq 1,
$$

then $\mathbf{x}^{*}$ defines the optimal strategy for portfolio liquidation in the presence of call auctions. An illustration is given in Figure 3.
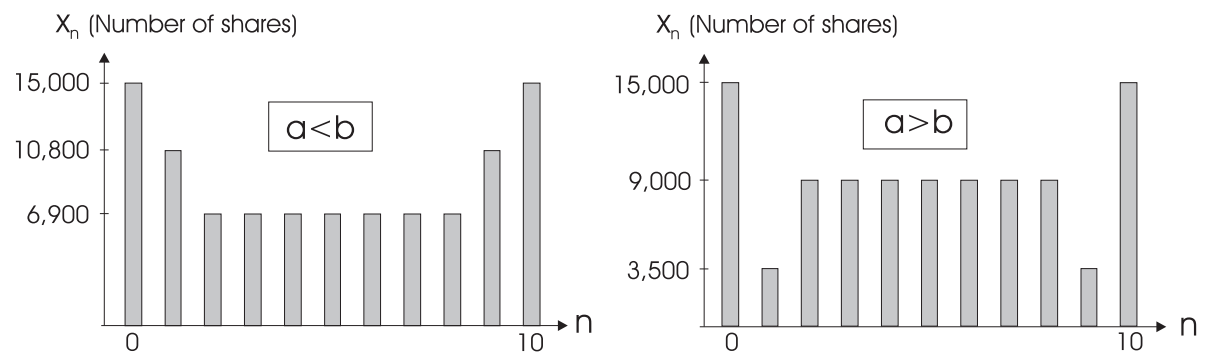

Fig. 3. Optimal strategies $\left(x_{0}^{*}, \ldots, x_{N}^{*}\right)$ in a market with call auctions: the large investor wants to buy $X_{0}=100,000$ shares of which at least $30 \%$ should be placed during call auctions $(\alpha=0.3)$. The resilience coefficients are $a=e^{-5}$ and $b=e^{-1}$ in the first graph and $a=e^{-1}$ and $b=e^{-5}$ in the second graph.

5. Proof of Theorem 3.3. We have to reduce the minimization of the cost functional

$$
\mathcal{C}(\xi)=\mathbb{E}\left[\sum_{n=0}^{N} \pi_{t_{n}}\left(\xi_{n}\right)\right]
$$

with respect to all admissible strategies $\xi$ to the minimization of the quadratic form $C(\mathbf{x}):=\frac{1}{2}\langle\mathbf{x}, M \mathbf{x}\rangle, \mathbf{x} \in \mathbb{R}^{N+1}$.

To this end, we first introduce simplified model dynamics by collapsing the bid-ask spread into a single value. More precisely, for any admissible strategy $\xi$, we define the process

$$
D_{t}:=D_{t}^{A}+D_{t}^{B}
$$

We now introduce the simplified price of $\xi_{n}$ at time $t_{n}$ by

$$
\bar{\pi}_{t_{n}}\left(\xi_{n}\right):=A_{t_{n}}^{0} \xi_{n}+\frac{q}{2}\left(D_{t_{n}+}^{2}-D_{t_{n}}^{2}\right),
$$

regardless of the sign of $\xi_{n}$. We have the following simple lemma.

LEMMA 5.1. For any admissible strategy $\xi$,

$$
\bar{\pi}_{t_{n}}\left(\xi_{n}\right) \leq \pi_{t_{n}}\left(\xi_{n}\right) \quad \text { with equality if } \xi_{k} \geq 0 \text { for all } k \leq n .
$$


Proof. Since $D_{t_{n}+}-D_{t_{n}}=\xi_{n} / q$, we can rewrite $\bar{\pi}_{t_{n}}\left(\xi_{n}\right)$ as

$$
\bar{\pi}_{t_{n}}\left(\xi_{n}\right)=\frac{q}{2}\left(\left(A_{t_{n}}^{0}+D_{t_{n}+}\right)^{2}-\left(A_{t_{n}}^{0}+D_{t_{n}}\right)^{2}\right) .
$$

If $\xi_{n} \geq 0$ then $D_{t_{n}+}=D_{t_{n}+}^{A}+D_{t_{n}+}^{B}=D_{t_{n}+}^{A}+D_{t_{n}}^{B}$, and hence

$$
\begin{aligned}
\bar{\pi}_{t_{n}}\left(\xi_{n}\right) & =\frac{q}{2}\left(\left(A_{t_{n}}^{0}+D_{t_{n}+}^{A}+D_{t_{n}}^{B}\right)^{2}-\left(A_{t_{n}}^{0}+D_{t_{n}}^{A}+D_{t_{n}}^{B}\right)^{2}\right) \\
& \leq \frac{q}{2}\left(\left(A_{t_{n}}^{0}+D_{t_{n}+}^{A}\right)^{2}-\left(A_{t_{n}}^{0}+D_{t_{n}}^{A}\right)^{2}\right) \\
& =\pi_{t_{n}}\left(\xi_{n}\right),
\end{aligned}
$$

due to the fact that $D_{t_{n}}^{B} \leq 0$.

If $\xi_{n} \leq 0$ then (3) and $D_{t_{n}+}^{B}-D_{t_{n}}^{B} \leq 0$ imply that

$$
\begin{aligned}
\bar{\pi}_{t_{n}}\left(\xi_{n}\right) & =\frac{q}{2}\left(\left(A_{t_{n}}+D_{t_{n}+}^{B}\right)^{2}-\left(A_{t_{n}}+D_{t_{n}}^{B}\right)^{2}\right) \\
& \leq \frac{q}{2}\left(\left(B_{t_{n}}^{0}+D_{t_{n}+}^{B}\right)^{2}-\left(B_{t_{n}}^{0}+D_{t_{n}}^{B}\right)^{2}\right)=\pi_{t_{n}}\left(\xi_{n}\right) .
\end{aligned}
$$

We now define a simplified price functional as

$$
\overline{\mathcal{C}}(\xi):=\mathbb{E}\left[\sum_{n=0}^{N} \bar{\pi}_{t_{n}}\left(\xi_{n}\right)\right]
$$

We will show that the simplified price functional $\overline{\mathcal{C}}$ has a unique minimizer $\xi^{*}$, which, if all its trades are nonnegative, must be the optimal strategy according to Lemma 5.1. To this end, we further reduce the minimization of $\overline{\mathcal{C}}$ to the minimization of a functional $\bar{C}$ defined on deterministic strategies. Let us use the notation

$$
X_{t}:=X_{0}-\sum_{t_{k}<t} \xi_{k} \text { for } t \leq T \text { and } X_{t_{N+1}}:=0 .
$$

The accumulated simplified price of an admissible strategy $\xi$ is

$$
\sum_{n=0}^{N} \bar{\pi}_{t_{n}}\left(\xi_{n}\right)=\sum_{n=0}^{N} A_{t_{n}}^{0} \xi_{n}+\frac{q}{2} \sum_{n=0}^{N}\left(D_{t_{n}+}^{2}-D_{t_{n}}^{2}\right)
$$

Integrating by parts yields

$$
\sum_{n=0}^{N} A_{t_{n}}^{0} \xi_{n}=-\sum_{n=0}^{N} A_{t_{n}}^{0}\left(X_{t_{n+1}}-X_{t_{n}}\right)=X_{0} A_{0}+\sum_{n=1}^{N} X_{t_{n}}\left(A_{t_{n}}^{0}-A_{t_{n-1}}^{0}\right) .
$$

Since $\xi$ is admissible, $X_{t}$ is a bounded predictable process. Hence, due to the martingale property of the unaffected best ask process $A^{0}$, the expectation of $(24)$ is hence equal to $X_{0} A_{0}$. Next, observe that the simplified extra spread process $D$ evolves deterministically once the values $\xi_{0}, \xi_{1}(\omega), \ldots, \xi_{N}(\omega)$ are given. Consequently, there exists a deterministic function $\bar{C}: \mathbb{R}^{N+1} \rightarrow \mathbb{R}$ such that

$$
\frac{q}{2} \sum_{n=0}^{N}\left(D_{t_{n}+}^{2}-D_{t_{n}}^{2}\right)=\bar{C}\left(\xi_{0}, \ldots, \xi_{N}\right) \text {. }
$$

It follows that

$$
\overline{\mathcal{C}}(\xi)=A_{0} X_{0}+\mathbb{E}\left[\bar{C}\left(\xi_{0}, \ldots, \xi_{N}\right)\right]
$$


We will now turn to further simplifying the function $\bar{C}$. For any deterministic strategy $\left(x_{0}, \ldots, x_{N}\right) \in \Xi$, the extra spread is given by $D_{t_{n}+}=D_{t_{n}}+x_{n} / q$ and

$$
D_{t}=\gamma \sum_{t_{n}<t} x_{n}+\kappa \sum_{t_{n}<t} e^{-\int_{t_{n}}^{t} \rho_{s} d s} x_{n} .
$$

Hence,

$$
\begin{aligned}
\bar{C}\left(x_{0}, \ldots, x_{N}\right) & =\frac{q}{2} \sum_{n=0}^{N}\left(D_{t_{n}+}^{2}-D_{t_{n}}^{2}\right)=\frac{q}{2} \sum_{n=0}^{N}\left(\left(D_{t_{n}}+x_{n} / q\right)^{2}-D_{t_{n}}^{2}\right) \\
& =\sum_{n=0}^{N} x_{n} D_{t_{n}}+\frac{1}{2 q} \sum_{n=0}^{N} x_{n}^{2} \\
& =\gamma \sum_{n=0}^{N} \sum_{k=0}^{n-1} x_{n} x_{k}+\kappa \sum_{n=0}^{N} \sum_{k=0}^{n-1} x_{n} e^{-\int_{t_{k}}^{t_{n}} \rho_{s} d s} x_{k}+\frac{\gamma+\kappa}{2} \sum_{n=0}^{N} x_{n}^{2} \\
& =\frac{\gamma}{2} X_{0}^{2}+\kappa \sum_{n=0}^{N} \sum_{k=0}^{n-1} x_{n} e^{-\int_{t_{k}}^{t_{n}} \rho_{s} d s} x_{k}+\frac{\kappa}{2} \sum_{n=0}^{N} x_{n}^{2} .
\end{aligned}
$$

Therefore it is enough to minimize the function

$$
C\left(x_{0}, \ldots, x_{N}\right):=\sum_{n=0}^{N} \sum_{k=0}^{n-1} x_{n} e^{-\int_{t_{k}}^{t_{n}} \rho_{s} d s} x_{k}+\frac{1}{2} \sum_{n=0}^{N} x_{n}^{2}
$$

over $\Xi_{0}$. Moreover, the problem is in fact independent of $\gamma$ and $\kappa$ as long as $\kappa>0$. Let us define a matrix $M$ by

$$
M_{i j}=e^{-\left|\int_{t_{i}}^{t_{j}} \rho_{s} d s\right|} \quad \text { for } i, j \in\{0, \ldots, N\} .
$$

Then

$$
C\left(x_{0}, \ldots, x_{N}\right)=C(\mathbf{x})=\frac{1}{2}\langle\mathbf{x}, M \mathbf{x}\rangle \quad \text { for } \mathbf{x}=\left(x_{0}, \ldots, x_{N}\right) \in \mathbb{R}^{N+1} .
$$

The matrix $M$ is symmetric and $C$ is a quadratic form. By recalling the notation (8), we obtain the representation (15) for $M$. We will show in the next section that $M$ is positive definite. From this, existence and uniqueness of a minimizer $\mathbf{x}^{*}$ of $C$ on $\Xi_{0}$ follow. By the preceding reduction arguments, we must then have $\xi^{*}=\mathbf{x}^{*}$, provided that all coordinates of $\mathbf{x}^{*}$ are nonnegative.

6. Proof of Theorem 3.4. Let $\mathbf{e}_{0}, \ldots, \mathbf{e}_{N}$ denote the canonical basis of $\mathbb{R}^{N+1}$ and let us define a set of vectors $\mathbf{y}_{0}, \ldots, \mathbf{y}_{N} \in \mathbb{R}^{N+1}$ by the following recursive formula:

$$
\begin{aligned}
\mathbf{y}_{0} & =\mathbf{e}_{0}, \\
\mathbf{y}_{n} & =\mathbf{y}_{n-1} a_{n}+\mathbf{e}_{n} \sqrt{1-a_{n}^{2}}, \quad n=1, \ldots, N .
\end{aligned}
$$

Then $M$ is equal to the corresponding Gram matrix, that is, $M_{i j}=\left\langle\mathbf{y}_{i}, \mathbf{y}_{j}\right\rangle$ for all pairs $i, j$. Indeed, induction first shows that $\left\langle\mathbf{y}_{i}, \mathbf{y}_{i}\right\rangle=1$ for all $i$, and then one readily obtains that $\left\langle\mathbf{y}_{i}, \mathbf{y}_{j}\right\rangle=\left\langle\mathbf{y}_{i}, \mathbf{y}_{i}\right\rangle a_{i+1} \cdots a_{j}=M_{i j}$ for $i<j$. It is also not difficult to show 
the following explicit formula for $\mathbf{y}_{n}$

$$
\mathbf{y}_{n}=\sum_{j=0}^{n}\left[\left(\prod_{i=j+1}^{n} a_{i}\right) \sqrt{1-a_{j}^{2}}\right] \mathbf{e}_{j},
$$

where we use the conventions $a_{0}:=0$ and $\prod_{i \in \emptyset}(\ldots)=1$. Let us denote by $Y$ the upper triangular matrix with columns $\mathbf{y}_{0}, \mathbf{y}_{1}, \ldots, \mathbf{y}_{N}$. Then

$$
M=Y^{\prime} Y \text {. }
$$

Since $t_{n}-t_{n-1}>0$ and $\rho_{t}>0$, we have $0<a_{n}<1$ for $n=1, \ldots, N$. Hence, the diagonal coefficients of the upper triangular matrix $Y$ are all strictly positive, and it follows that $Y$ is invertible. Consequently,

$$
C\left(x_{0}, \ldots, x_{N}\right)=\frac{1}{2} \mathbf{x}^{\prime} M \mathbf{x}=\frac{1}{2}\|Y \mathbf{x}\|^{2}>0 \quad \text { for all nonzero } \mathbf{x}=\left(x_{0}, \ldots, x_{N}\right)^{\prime} .
$$

In particular, $M$ is positive definite as claimed in Theorem 3.3.

Note that the gradient of $C$ at $\mathbf{x}$ is given by $M \mathbf{x}$. Hence, under the assumptions of the theorem, the Kuhn-Tucker theorem, e.g., in the form given in Borwein and Lewis [8, Theorem 7.2.9], states the existence of multipliers $\lambda_{0} \lambda_{1}, \ldots, \lambda_{k} \in \mathbb{R}$ and $\mu_{j} \geq 0, j \in J$, such that

$$
M \mathbf{x}^{*}=\lambda_{0} \mathbf{1}+\sum_{i=1}^{k} \lambda_{i} \mathbf{u}^{i}+\sum_{j \in J} \mu_{j} \mathbf{v}^{j} .
$$

Multiplication with $M^{-1}$ gives (13). Since $\{\mathbf{1}\} \cup\left\{\mathbf{u}^{1}, \ldots, \mathbf{u}^{k}\right\} \cup\left\{\mathbf{v}^{j} \mid j \in J\right\}$ is a set of linearly independent vectors in $\mathbb{R}^{N+1}$, it is clear that the multipliers are uniquely determined by (14).

Let us now prove the formula for $M^{-1}$. By (27), we have

$$
Y^{\prime}=\left[\begin{array}{ccccc}
1 & 0 & \cdots & \cdots & 0 \\
a_{1} & \sqrt{1-a_{1}^{2}} & 0 & \cdots & 0 \\
\vdots & \ddots & \ddots & \ddots & \vdots \\
a_{1} \cdots a_{N-1} & \cdots & & \sqrt{1-a_{N-1}^{2}} & 0 \\
a_{1} \cdots a_{N} & \cdots & & a_{N} \sqrt{1-a_{N-1}^{2}} & \sqrt{1-a_{N}^{2}}
\end{array}\right] .
$$

To invert the matrix $Y^{\prime}$, we write the equation $Y^{\prime} \mathbf{z}=\mathbf{x}$ for $\mathbf{z}=\left(z_{0}, \ldots, z_{N}\right)^{\prime}$ and $\mathbf{x}=\left(x_{0}, \ldots, x_{N}\right)^{\prime}$ in the following way:

$$
\begin{aligned}
& z_{0}=x_{0}, \\
& a_{1} z_{0}+\sqrt{1-a_{1}^{2}} z_{1}=x_{1}, \\
& a_{1} a_{2} z_{0}+a_{2} \sqrt{1-a_{1}^{2}} z_{1}+\sqrt{1-a_{2}^{2}} z_{2}=x_{2}, \\
& \cdots \\
&\left(a_{1} \cdots a_{N}\right) z_{0}+\left(a_{2} \cdots a_{N}\right) \sqrt{1-a_{1}^{2}} z_{1}+\cdots+\sqrt{1-a_{N}^{2}} z_{N}=x_{N} .
\end{aligned}
$$


This can be simplified as follows:

$$
\begin{aligned}
z_{0} & =x_{0}, \\
a_{1} x_{0}+\sqrt{1-a_{1}^{2}} z_{1} & =x_{1}, \\
a_{2} x_{1}+\sqrt{1-a_{2}^{2}} z_{2} & =x_{2}, \\
& \cdots \\
a_{N} x_{N-1}+\sqrt{1-a_{N}^{2}} z_{N} & =x_{N} .
\end{aligned}
$$

We therefore get

$$
\left(Y^{\prime}\right)^{-1}=\left[\begin{array}{ccccc}
1 & 0 & \cdots & \cdots & 0 \\
\frac{-a_{1}}{\sqrt{1-a_{1}^{2}}} & \frac{1}{\sqrt{1-a_{1}^{2}}} & 0 & \cdots & 0 \\
0 & \ddots & \ddots & 0 & 0 \\
\vdots & \ddots & \frac{-a_{N-1}}{\sqrt{1-a_{N-1}^{2}}} & \frac{1}{\sqrt{1-a_{N-1}^{2}}} & 0 \\
0 & \cdots & 0 & \frac{-a_{N}}{\sqrt{1-a_{N}^{2}}} & \frac{1}{\sqrt{1-a_{N}^{2}}}
\end{array}\right]
$$

It follows that $M^{-1}=Y^{-1}\left(Y^{\prime}\right)^{-1}$ is given by the following tridiagonal matrix:

$$
M^{-1}=\left[\begin{array}{ccccc}
\frac{1}{1-a_{1}^{2}} & \frac{-a_{1}}{1-a_{1}^{2}} & 0 & \cdots & 0 \\
\frac{-a_{1}}{1-a_{1}^{2}} & \left(\frac{1}{1-a_{1}^{2}}+\frac{a_{2}^{2}}{1-a_{2}^{2}}\right) & \frac{-a_{2}}{1-a_{2}^{2}} & 0 \cdots & 0 \\
0 & \ddots & \ddots & \ddots & \vdots \\
\vdots & \ddots & \frac{-a_{N-1}}{1-a_{N-1}^{2}} & \left(\frac{1}{1-a_{N-1}^{2}}+\frac{a_{N}^{2}}{1-a_{N}^{2}}\right) & \frac{-a_{N}}{1-a_{N}^{2}} \\
0 & \cdots & 0 & \frac{-a_{N}}{1-a_{N}^{2}} & \frac{1}{1-a_{N}^{2}}
\end{array}\right] .
$$

This concludes the proof of Theorem 3.4.

Acknowledgements. The authors thank the Quantitative Products Group of Deutsche Bank, in particular Marcus Overhaus, Hans Bühler, Andy Ferraris, Alexander Gerko, and Chrif Youssfi for stimulating discussions and useful comments on optimal portfolio liquidation (the statements in this paper, however, express the private opinion of the authors and do not necessarily reflect the views of Deutsche Bank).

\section{References}

[1] A. Alfonsi, A. Schied and A. Fruth, Optimal execution strategies in limit order books with general shape functions, preprint, TU Berlin, 2007.

[2] R. Almgren, Optimal execution with nonlinear impact functions and trading-enhanced risk, Applied Mathematical Finance 10 (2003), 1-18.

[3] R. Almgren and N. Chriss, Value under liquidation, Risk (1999).

[4] R. Almgren and N. Chriss, Optimal execution of portfolio transactions, J. Risk 3 (2000), $5-39$. 
[5] R. Almgren and J. Lorenz, Adaptive arrival price, in: Algorithmic Trading III: Precision, Control, Execution, Brian R. Bruce (ed.), Institutional Investor Journals (2007).

[6] D. Bertsimas and A. Lo, Optimal control of execution costs, Journal of Financial Markets 1 (1998), 1-50.

[7] B. Biais, P. Hillion and C. Spatt, An empirical analysis of the limit order book and order flow in Paris Bourse, Journal of Finance 50 (1995), 1655-1689.

[8] J. Borwein and A. Lewis, Convex Analysis and Nonlinear Optimization. Theory and Examples, 2nd ed., CMS Books in Mathematics/Ouvrages de Mathématiques de la SMC, 3, Springer, New York, 2006.

[9] J. P. Bouchaud, Y. Gefen, M. Potters and M. Wyart, Fluctuations and response in financial markets: the subtle nature of 'random' price changes, Quantitative Finance 4 (2004), 176.

[10] M. Brunnermeier and L. Pedersen,. Predatory trading, Journal of Finance 60 (2005), $1825-1863$.

[11] B. Carlin, M. Lobo and S. Viswanathan, Episodic liquidity crises: Cooperative and predatory trading, Journal of Finance (2007).

[12] C. Kehr, J. Krahnen and E. Theissen, The anatomy of a call market, J. Financial Intermediation 10 (2001), 249-270.

[13] A. Obizhaeva and J. Wang, Optimal trading strategy and supply/demand dynamics, Journal of Financial Markets (2006).

[14] M. Potters and J.-P. Bouchaud, More statistical properties of order books and price impact, Physica A 324 (2003), 133-140.

[15] A. Schied and T. Schöneborn, Optimal basket liquidation with finite time horizon for CARA investors, preprint, Cornell University and TU Berlin, 2007.

[16] A. Schied and T. Schöneborn, Risk aversion and the dynamics of optimal liquidation strategies in illiquid markets, preprint, Cornell University and TU Berlin, 2008.

[17] T. Schöneborn and A. Schied, Liquidation in the face of adversity: stealth vs. sunshine trading, predatory trading vs. liquidity provision, preprint, Cornell University and TU Berlin, 2007.

[18] P. Weber and B. Rosenow, Order book approach to price impact, Quantitative Finance 5 (2005), 357-364. 
